\title{
8 Research Square

\section{Understanding the Impact of Histology on the Post- Surgery Prognosis for Patients 70 Years and Older With I A Stage Non-Small Lung Cancer}

\section{Bo Hao}

Renmin Hospital of Wuhan University: Wuhan University Renmin Hospital

Hao Jie Feng

Renmin Hospital of Wuhan University: Wuhan University Renmin Hospital

Xiao Xia Wan

Ezhou Central Hospital

\section{Lin Zhang}

Renmin Hospital of Wuhan University: Wuhan University Renmin Hospital

\section{Zi Long Lu}

Renmin Hospital of Wuhan University: Wuhan University Renmin Hospital

\section{Bohao Liu}

Renmin Hospital of Wuhan University: Wuhan University Renmin Hospital

Ru Yuan He

Renmin Hospital of Wuhan University: Wuhan University Renmin Hospital

Heng Meng

Renmin Hospital of Wuhan University: Wuhan University Renmin Hospital

\section{Tao Fan}

Renmin Hospital of Wuhan University: Wuhan University Renmin Hospital

\section{Wen Yang Jiang}

Renmin Hospital of Wuhan University: Wuhan University Renmin Hospital

Qing Geng ( $\sim$ gengqingwhu@whu.edu.cn )

Renmin Hospital of Wuhan University: Wuhan University Renmin Hospital

\section{Research}

Keywords: Lung adenocarcinoma, lung squamous cell carcinoma, sublobar resection, lobectomy

Posted Date: December 16th, 2020

DOI: https://doi.org/10.21203/rs.3.rs-127800/v1 
License: (c) (i) This work is licensed under a Creative Commons Attribution 4.0 International License. Read Full License 


\section{Abstract}

Objectives: Few studies investigated whether the types of histology had an influence on the postsurgical prognosis for patients 70 years and older with $\otimes \mathrm{A}$ stage non-small lung cancer (NSCLC).

Methods: The study population in our study were obtained from the Surveillance, Epidemiology, and End Results (SEER) Program.

Results: A total of 10,376 eligible patients were included in our study. In both lung adenocarcinoma (LUAD) and lung squamous cell carcinoma (LSQCC) patients with TS $\leq 10 \mathrm{~mm}$, sublobar resection (SR) achieved similar overall survival (OS) (LUAD: HR=0.843, 95\% Cl $(0.673,1.062), \mathrm{P}=0.149$; LSQCC: $\mathrm{HR}=0.799,95 \% \mathrm{Cl}(0.615,0.036), \mathrm{P}=0.091)$ and LCSS (LUAD: HR=1.074, 95\% $\mathrm{Cl}(0.626,1.843), \mathrm{P}=0.795$; LSQCC: HR=0.987, $95 \% \mathrm{Cl}(0.532,1.833), \mathrm{P}=0.967)$ to lobectomy (LT). For LUAD patients with $T S>10 \mathrm{~mm}$ $\& \leq 20 \mathrm{~mm}$, LT was associated with better OS ( $\mathrm{HR}=0.785,95 \% \mathrm{Cl}(0.703,878), \mathrm{P}<0.001)$ but not LCSS ( $\mathrm{HR}=0.962,95 \% \mathrm{Cl}(0.766,1.209), \mathrm{P}=0.741)$; while for $\mathrm{LSQCC}$, $\mathrm{LT}$ yielded similar OS $(\mathrm{HR}=0.864,95 \% \mathrm{Cl}$ $(0.746,1.005), P=0.051)$ and LCSS $(H R=0.872,95 \% \mathrm{Cl}(0.675,1.128), P=0.297)$. LUAD patients with TS $>20$ $\mathrm{mm} \& \leq 30 \mathrm{~mm}$ received $\mathrm{SR}$ were at a significant risk of reduction of $\mathrm{OS}(\mathrm{HR}=0.816,95 \% \mathrm{Cl}(0.709,0.938)$, $\mathrm{P}=0.004)$ but not LCSS $(\mathrm{HR}=0.954,95 \% \mathrm{Cl}(0.732,1.244), \mathrm{P}=0.729)$; while for LSQCC, patients received LT had a better OS $(H R=0.742,95 \% \mathrm{Cl}(0.624,0.883), \mathrm{P}<0.001)$ but not LCSS $(\mathrm{HR}=0.776,95 \% \mathrm{Cl}(0.563$, 1.071), $P=0.123)$.

Conclusion: The postsurgical prognosis differed in patients with different histology, and SR maybe acceptable for elderly LUAD patients with TS $\leq 10 \mathrm{~mm}$ and LSQCC patients with TS $\leq 20 \mathrm{~mm}$.

\section{Introduction}

Non-small lung cancer (NSCLC) including lung squamous cell carcinoma (LSQCC) and adenocarcinoma (LUAD) constitute approximately $85 \%$ of lung cancer cases[1]. Although great advances have been achieved, the survival of lung cancer patients still remains poor. In earlier years, LSQCC was more prevalent than LUAD; while in recent years, LUAD became the most common histologic type[2]. Several years ago, there were some researches demonstrating that the prognosis of LUAD was similar to that of LSQCC[3-5]. However, recent studies pointed out that some crucial prognostic factors and prognosis are quite different between those two types of lung cancers, and that they should be differently analyzed[5, 6]. Wang et al. [5] found that LUAD was significant different from LSQCC in gender, location, age, smoking status and survival; LUAD was more common in younger populations compared to LSQCC; moreover, LUAD patients were likely to have favorable long-term outcome than that of LSQCC patients in all tumor stage. Therefore, the postsurgical outcomes of LUAD and LSQCC need to be well researched.

Currently, it is widely acknowledged that surgical resection might be the curative treatment for early-stage NSCLC. However, controversy still remains about whether sublobar resection (SR) could achieve a similar outcome to lobectomy (LT) for early-stage NSCLC. Moreover, patients with an order age received pulmonary resection were likely to be associated with higher risks of perioperative morbidities and 
mortality rates, worse cardiopulmonary function and shorter life expectancy [7, 8]. As mentioned, LUAD and LSQCC were different in gender, age, smoking status and survival. Thus, whether histology had a significant influence on postsurgical outcome of elderly patients with early-stage NSCLC? Despite the fact that numerous studies have investigated the impact of histology on survival, these studies were mainly focused on other prognostic factors than surgery and didn't deeply and comprehensively investigate into the relationship between histology and prognosis. Therefore, in the present study, the clinical data from the Surveillance, Epidemiology, and End Results (SEER) database were collected to investigate the prognostic significance of histology on survival of patients who underwent surgery.

\section{Material And Methods}

\section{The study population}

Eligible patients were selected from the SEER database which was funded by the National Cancer Institute of the United States. The database provides the cancer incidence and survival data which were collected from several registries that covers more than $30 \%$ of the U.S. population. And the clinical data have been collected for numerous high-quality clinical studies[9-14].

The inclusion criteria in our study include: a) pathologically confirmed primary T1NOMO NSCLC, only LUAD and LSQCC with a tumor size(TS) $\leq 30 \mathrm{~mm}$; b) no history of chemotherapy before or after surgery; c) history of surgery, excluding extended lobectomy, pneumonectomy and extended pneumonectomy; d) no record of radiation treatment before or after surgery; e) tumor was not located in main bronchus; $f$ ) age $>18 ; g)$ Only active follow-up was included; $h$ ) survival of less than 3 months after the operation was excluded.

Using SEER data from 1 Jan 2004 to 31 Dec 2011, we collected available data including the demographic information (age at diagnosis, sex, race, marriage status), tumor details (tumor size, histology, differentiation grade, TNM stage, location, and literally, the number of resected regional lymph nodes), treatment records (surgical procedures, radiation, chemotherapy) and predictors of survival (OS and lung cancer specific survival (LCSS)).

The histologic types of all enrolled cases were identified according to the 3th edition of International Classification of Diseases for Oncology (ICD-0-3). The histologic types were included as follows: adenocarcinoma (8140-8141, 8200, 8250-8255, 8470, 8480-8481, 8550-8551, 8570-8573) and squamous cell carcinoma $(8052,8070-8076,8078,8083-8084)$ [15-17]. The whole cohort was divided into two cohorts according to the types of histology (LUAD and LSQCC). The marriage status was divided into married, unmarried (including divorced, separated, single and widowed) and others group(unknown). Surgical procedures (SP) were divided into SR (surgery code: 21,22 ) and standard LT (surgery code :30, 33). SR was comprised of wedge resection and segmentectomy. Grade well/moderate group included grade $\nabla$ and $\nabla$, and poor/undifferentiated one included $\nabla$ and $\nabla$.

\section{Statistical analysis}


In our study, the primary endpoints were OS and LCSS. The length of time from diagnosis to the last follow up or individuals' death of any cause was defined as the OS. The length of time from diagnosis to the last follow up or individuals' death due to lung cancer was defined as LCSS. The last update on the follow-up time was Dec 31, 2016. OS and LCSS were assessed by Kaplan-Meier analysis. Log-rank tests were used to estimate survival differences between two types of histology. Multivariate analyses were conducted to remove biases as many as possible (Only when univariate analysis indicted a significant statistical difference, multivariate analysis was conducted).

Pearson's $\chi 2$ tests were used for categorical variables and the Wilcoxon tests were used for continuous variables. R 3.6.1 (R Development Core Team, Vienna, Austria) was used to establish Kaplan-Meier survival curves in our study. Univariate and multivariate Cox-regression analyses was performed to calculate hazard ratios (HRs) and its 95\% confidence intervals (Cls) using SPSS 22.0 (IBM, Armonk, NY). All statistical analyses were two-sided, and $P$ value less than 0.05 revealed a statistically significance.

\section{Results}

\section{Baseline characteristics}

As shown in Table 1, a total of 10,376 NSCLC patients (only adenocarcinoma and squamous cell carcinoma) were included. Among them, 7,197 patients were diagnosed with LUAD and 3,179 with LSQCC. The full cohort was categorized into adenocarcinoma and squamous cell carcinoma cohort. In LSQCC patients, 1048 received SR and 2131 received LT; while for LUAD, 2248 received SR and 4949 received LT. In both LUAD and LSQCC, sublobar resection was likely to be performed in patients with a smaller TS and was also associated with a smaller number of resected lymph nodes. More details were available in Table 1. 
Table 1

Baseline characteristics the study population

Squamous cell carcinoma $(n=3179)$

$\begin{array}{ll}\begin{array}{l}\text { Sublobar } \\ \text { resection }\end{array} & \text { Lobectomy } \\ (n=1048) & (n=2131)\end{array}$

Sex

Male

Female

Race

White

Black

Others

Grade

Well/moderate

Poor/UD

Unknown

Resected LNs

0

$1-3$

$\geq 4$

Unknown

Tumor size

(mm)

$\leq 10$

$11-20$

$21-30$
544(51.9\%) 1130(53.0\%)

504(48.1\%) 1001(47.0\%)

0.872

959(91.5\%) 1946(91.3\%)

$59(5.6 \%) \quad 117(5.5 \%)$

$0.045^{\star}$
$30(2.9 \%) \quad 68(3.2 \%)$
Adenocarcinoma $(n=7197)$

Sublobar Lobectomy

resection

$(n=2248)$

$(n=4949)$

0.553

0.659

950(42.3\%) 2064(41.7\%)

1298(57.7\%) 2885(58.3\%)

$0.043^{*}$

$2037(90.6 \%) \quad 4398(88.7 \%)$

$90(4.0 \%) \quad 800(4.6 \%)$

$121(5.4 \%) \quad 846(6.7 \%)$

$<$

$0.001^{*}$

1628(72.4\%) 3755(75.9\%)

$400(17.8 \%) \quad 870(17.6 \%)$

$220(9.8 \%) \quad 324(6.5 \%)$
$51(4.9 \%) \quad 66(3.1 \%)$

<.001*

527(50.3\%) 84(3.9\%)

$252(24.0 \%) \quad 395(18.5 \%)$

221(21.1\%) 1545(72.5\%)

$48(4.6 \%) \quad 107(5.0 \%)$

$<$

$0.001^{\star}$

$153(14.6 \%) \quad 174(8.2 \%)$

602(57.4\%) 1012(47.5\%)

$293(28.0 \%) \quad 945(44.3 \%)$

\begin{tabular}{|c|c|c|}
\hline & $1082(48.1 \%)$ & 192(3.9\%) \\
\hline & $556(24.7 \%)$ & $876(17.7 \%)$ \\
\hline & $513(22.8 \%)$ & $3611(73.0 \%)$ \\
\hline & $97(4.3 \%)$ & $270(5.5 \%)$ \\
\hline \multicolumn{3}{|l|}{$\begin{array}{l}< \\
0.001 *\end{array}$} \\
\hline & $470(20.9 \%)$ & $408(8.2 \%)$ \\
\hline & $1260(56.0 \%)$ & $2507(50.7 \%)$ \\
\hline & $518(23.0 \%)$ & $2034(41.1 \%)$ \\
\hline
\end{tabular}

$<$

$0.001^{*}$

* indicates that the difference was statistically significant.

Abbreviations: UD, undifferentiated; LN, lymph node. 


\section{Squamous cell carcinoma $(n=3179)$}

$0.012^{*}$

Adenocarcinoma( $\mathbf{n}=7197)$

\begin{tabular}{|c|c|c|c|c|c|c|}
\hline $\begin{array}{l}\text { Marriage } \\
\text { status }\end{array}$ & & & $0.012 *$ & & & $0.006 *$ \\
\hline Married & $883(52.2 \%)$ & $2308(56.5 \%)$ & & $1209(53.8 \%)$ & 2858(57.7\%) & \\
\hline Unmarried & $747(44.2 \%)$ & $1645(40.3 \%)$ & & $956(42.5 \%)$ & 1934(39.1\%) & \\
\hline others & $61(3.6 \%)$ & 133(3.3\%) & & $83(3.7 \%)$ & $157(3.2 \%)$ & \\
\hline Location & & & 0.298 & & & <. $0.001 *$ \\
\hline Upper & $645(61.5 \%)$ & $1318(61.8 \%)$ & & $1304(58.0 \%)$ & $3048(61.6 \%)$ & \\
\hline Lower & $357(34.1 \%)$ & 694(32.6\%) & & $833(37.1 \%)$ & 1524(30.8\%) & \\
\hline others & $46(4.4 \%)$ & $119(5.6 \%)$ & & $111(4.9 \%)$ & $377(7.6 \%)$ & \\
\hline Laterality & & & $0.037 *$ & & & <. \\
\hline Left & 493(47.0\%) & $919(43.1 \%)$ & & $1039(46.2 \%)$ & 1912(38.6\%) & \\
\hline Right & $555(53.0 \%)$ & $1212(56.9 \%)$ & & $1209(53.8 \%)$ & $3037(61.4 \%)$ & \\
\hline
\end{tabular}

* indicates that the difference was statistically significant.

Abbreviations: UD, undifferentiated; LN, lymph node.

\section{Survival analyses for overall survival and lung cancer specific survival}

As shown in Figs. 1 and 2, survival curves analyses showed that LT yielded better OS $(\mathrm{HR}=0.647,95 \% \mathrm{Cl}$ $(0.606,0.689), P<0.001)$ and LCSS $(H R=0.834,95 \% \mathrm{Cl}(0.731,0.951), P=0.007)$ than $S R$ in $\otimes A$ stage LUAD patients; while in LSQCC, LT yielded better OS (HR $=0.706,95 \% \mathrm{Cl}(0.649,0.769), \mathrm{P}<0.001)$ but not better LCSS $(\mathrm{HR}=0.884,95 \% \mathrm{Cl}(0.740,1.055), \mathrm{P}=0.171)$. To further confirm the results, multivariate coxregression analyses were performed. As shown in Tables 2 and 3, LT was associated with better OS (HR = $0.806,95 \% \mathrm{Cl}(0.743,0.873), \mathrm{P}<0.001)$ but not LCSS $(\mathrm{HR}=0.992,95 \% \mathrm{Cl}(0.841,1.170), \mathrm{P}=0.920)$ in patients with LUAD compared with SR. In patients with LSQCC, LT was associated with better OS (HR = $0.804,95 \% \mathrm{Cl}(0.722,0.895), \mathrm{P}<0.001)$ in patients compared with SR. In addition, we also found that male and a larger tumor size were closely associated with lower OS and LCSS in $\triangle$ A stage patients with LUAD or LSQCC. Interestingly, advanced tumor grade predicted worse OS (Multivariate: $\mathrm{HR}=1.492,95 \% \mathrm{Cl}(1.383$, 1.610), $\mathrm{P}<0.001$ ) and LCSS (Multivariate: $\mathrm{HR}=1.823,95 \% \mathrm{Cl}(1.582,2.101), \mathrm{P}<0.001)$ in $\otimes \mathrm{A}$ stage patients with LUAD; while in LSQCC, the difference was not observed (Univariate: OS, $\mathrm{HR}=1.148,95 \% \mathrm{Cl}(0.944-$ 1.077), $P=0.806$; multivariate: $L C S S, H R=1.067,95 \% C l(0.942-1.209), P=0.307)$. The number of resected lymph nodes (LNs) was strongly related to the outcome of the patients. Both univariate and multivariate analyses showed that a larger number of resected LNs predicted shorter OS and LCSS in LUAD and LSQCC patients (Table 2, Table 3, Supplementary table 1 and Table 2). We also notice that female was 
associated with favorable OS and LCSS in LUAD; while in LSQCC, male was associated with worse OS but not LCSS. 
Table 2

Multivariate regression analyses for overall survival

\section{Adenocarcinoma}

$\mathrm{HR} \quad 95 \% \mathrm{Cl}$
Squamous cell carcinoma

$\mathrm{HR} \quad 95 \% \mathrm{Cl}$
$\mathrm{P}$

Sex

Male

1

Female

0.671

$0.628-0.717$

$<0.001^{\star}$

0.776

$0.715-0.842<0.001^{\star}$

\section{Marriage status}

Married

1

Unmarried

1.294

$1.209-1.384$

$<0.001^{*}$

Others

1.082

0.902-1.299

0.396

\section{Grade}

Well/moderate

1

Poor/UD

1.492

$1.383-1.610$

$<0.001^{\star}$

Unknown

0.997

$0.886-1.121$

0.959

\section{Resected LNs}

0

$1-3$

0.749

$0.678-0.828<0.001$ *

1

$\geq 4$

0.573

$0.520-0.631<0.001$ *

0.600

$0.513-0.703<0.001 *$

0.771

$0.673-0.882<0.001^{*}$

Unknown

0.600

Tumor size (mm)

$\leq 10$ 1

$11-20$

1.117

$1.009-1.237$

$0.032 *$

0.687

$0.603-0.783$

$<0.001^{*}$

21-30

1.300

$1.169-1.448$

$<0.001^{*}$

0.909

$0.741-1.115$

0.361

SP

SR

LT

0.806

$0.743-0.873<0.001^{*}$

0.804

$0.722-0.895$

$<0.001^{*}$

* indicates that the difference was statistically significant

Abbreviations: UD, undifferentiated; LN, lymph node; SP, surgical procedure; SR, sublobar resection; LT, lobectomy; SCC, squamous cell carcinoma. 
Table 3

Multivariate regression analyses for lung cancer specific survival

Adenocarcinoma

$\mathrm{HR} \quad 95 \% \mathrm{Cl}$
Squamous cell carcinoma

P $\quad \mathrm{HR} \quad 95 \% \mathrm{Cl} \quad \mathrm{P}$

Sex

Male

1

Female

0.778

$0.682-0.887$

$<0.001^{*}$

Marriage Status

Married

1

Unmarried

1.336

$1.171-1.525$

$<0.001^{*}$

Others

0.864

$0.578-1.291$

0.475

Grade

Well/moderate

1

$\begin{array}{lllllll}\text { Poor/UD } & 1.823 & 1.582-2.101 & <0.001 * & 1.148 & 0.971-1.357 & 0.107 \\ \text { Unknown } & 0.935 & 0.728-1.200 & 0.597 & 0.764 & 0.447-1.307 & 0.326\end{array}$

\section{Resected LNs}

0

1

1

$\begin{array}{lllllll}1-3 & 0.776 & 0.633-0.952 & 0.015^{*} & 0.828 & 0.636-1.078 & 0.161\end{array}$

$\geq 4$

$0.568 \quad 0.465-0.693<0.001^{*}$

$0.694 \quad 0.553-0.870$

$0.002^{*}$

Unknown

$0.556 \quad 0.401-0.770<0.001$ *

0.874

$0.576-1.328$

0.529

Tumor size(mm)

$\leq 10$

1

$11-20$

1.379

$1.098-1.733$

$0.006 *$

1.154

$1.000-1.331$

$0.049 *$

$21-30$

1.841

$1.456-2.329$

$<0.001^{*}$

1.328

$1.145-1.540$

$<0.001^{\star}$

SP

$\mathrm{SR}$

LT

1

$0.992 \quad 0.841-1.170$

0.920

* indicates that the difference was statistically significant

Abbreviations: UD, undifferentiated; LNs, lymph nodes; SCC, squamous cell carcinoma; SR, sublobar resection; LT, lobectomy; SP, surgical procedure. 


\section{Survival analyses for overall survival and lung cancer specific survival stratified by tumor size}

To investigate the impact of histology on survival in different-sized tumor, the study population was further analyzed. As shown in Supplementary Figs. 1 and 2, for patients with TS $\leq 10 \mathrm{~mm}$, SR achieved better OS in LUAD $(\mathrm{HR}=0.668,95 \% \mathrm{Cl}(0.555,0.804), \mathrm{P}<0.001)$ but not in LSQCC $(\mathrm{HR}=0.799,95 \% \mathrm{Cl}$ $(0.615,0.036), P=0.091)$, and similar LCSS in LUAD (HR $=0.866,95 \% \mathrm{Cl}(0.568,1.320), P=0.502)$ and LSQCC (HR $=0.987,95 \% \mathrm{Cl}(0.532,1.833), \mathrm{P}=0.967)$ compared with $\mathrm{LT}$; while for $\mathrm{TS}>20 \mathrm{~mm} \& \leq 30 \mathrm{~mm}$, LT yielded better OS (LUAD: HR $=0.645,95 \% \mathrm{Cl}(0.572,0.726), \mathrm{P}<0.001$; $\mathrm{LSQCC}$ : $\mathrm{HR}=0.627,95 \% \mathrm{Cl}(0.542$, $0.724), P<0.001)$ and LCSS (LUAD: $H R=0.738,95 \% \mathrm{Cl}(0.588,0.927), P=0.009 ; \mathrm{LSQCC}: \mathrm{R}=0.696,95 \% \mathrm{Cl}$ $(0.530,0.914), P=0.009)$ in LUAD and LSQCC compared with SR; For patients with TS $>10 \mathrm{~mm}$ $\& \leq 20 \mathrm{~mm}, \mathrm{LT}$ yielded longer OS $(\mathrm{HR}=0.595,95 \% \mathrm{Cl}(0.545,0.650), \mathrm{P}<0.001)$ and LCSS $(\mathrm{HR}=0.738$, $95 \% \mathrm{Cl}(0.588,0.927), \mathrm{P}=0.009)$ in LUAD, and better OS $(\mathrm{HR}=0.751,95 \% \mathrm{Cl}(0.626,0.900), \mathrm{P}=0.002)$ but not LCSS $(\mathrm{HR}=0.872,95 \% \mathrm{Cl}(0.675,1.128), \mathrm{P}=0.297)$ in LSQCC.

Due to relative reliable than univariate ones, multivariate analyses were also performed to evaluate outcomes of $\triangle A$ stage NSCLC patients. As shown in Tables 4 and 5 , in LUAD patients with TS $\leq 10 \mathrm{~mm}$, LT achieved similar OS ( $\mathrm{HR}=0.843,95 \% \mathrm{Cl}(0.673,1.062), \mathrm{P}=0.149)$ and $\mathrm{LCSS}(\mathrm{HR}=1.074,95 \% \mathrm{Cl}(0.626$, 1.843), $P=0.795)$ compared with $S R$. For adenocarcinoma with $T S>10 \mathrm{~mm} \& \leq 20 \mathrm{~mm}$, LT was associated with better OS ( $\mathrm{HR}=0.785,95 \% \mathrm{Cl}(0.703,878), \mathrm{P}<0.001)$ but not $\mathrm{LCSS}(\mathrm{HR}=0.962,95 \% \mathrm{Cl}$ $(0.766,1.209), P=0.741)$; while for LSQCC patients, $L T$ seemed to achieve better OS ( $H R=0.864,95 \% \mathrm{Cl}$ $(0.746,1.005), P=0.051)$ but not significant. LUAD patients with $T S>20 \mathrm{~mm} \& \leq 30 \mathrm{~mm}$ received $S R$ were at a significant risk of reduction of $\mathrm{OS}(\mathrm{HR}=0.816,95 \% \mathrm{Cl}(0.709,0.938), \mathrm{P}=0.004)$ and but not LCSS (HR $=0.954,95 \% \mathrm{Cl}(0.732,1.244), \mathrm{P}=0.729)$; while for LSQCC, patients received $\mathrm{LT}$ had a better OS $(\mathrm{HR}=$ $0.742,95 \% \mathrm{Cl}(0.624,0.883), \mathrm{P}<0.001)$ but not LCSS $(\mathrm{HR}=0.776,95 \% \mathrm{Cl}(0.563,1.071), \mathrm{P}=0.123)$. 
Table 4

Multivariate regression analyses for overall survival stratified by tumor size

Adenocarcinoma

$\mathrm{HR} \quad 95 \% \mathrm{Cl}$

Tumor size $\leq 10(\mathrm{~mm})$

\section{Gender}

Male

Female

Grade

Well/moderate

Poor/UD

Unknown

Resected LNs

0

$1-3$

$\geq 4$

Unknown

SP

SR

LT

$10<$ Tumor size $\leq 20$

\section{Gender}

Male

Female

Grade

Well/moderate

Poor/UD
1

$0.6130 .510-0.736<0.001$ *

1

$1.354 \quad 1.047-1.750 \quad 0.021$ *

$1.129 \quad 0.865-1.475 \quad 0.371$
Squamous cell carcinoma

$\mathrm{HR} \quad 95 \% \mathrm{Cl} \quad \mathrm{P}$



Unknown
$\begin{array}{lll}0.997 & 0.848-1.173 & 0.972\end{array}$

Resected LNs

0

$1-3$

1

1

$\geq 4$

$0.7560 .661-0.865<0.001^{*}$

0.792

$0.662-0.948$

$0.011^{\star}$

$0.5770 .506-0.659<0.001 *$

0.643

$0.539-0.767<0.001^{*}$

Unknown

$0.5020 .400-0.631<0.001 *$

0.693

$0.525-0.914$

$0.009 *$

\section{Marriage status}

\begin{tabular}{llll} 
Married & 1 & & \\
\hline Unmarried & 1.259 & $1.147-1.381$ & $<0.001$ \\
\hline Others & 1.199 & $0.940-1.529$ & 0.144 \\
\hline Race & & & \\
\hline White & 1 & & \\
\hline Black & 1.080 & $0.877-1.329$ & 0.470 \\
\hline Others & 0.842 & $0.694-1.021$ & 0.080 \\
\hline
\end{tabular}

SP

$\mathrm{SR}$

1

1

LT

0.785

$0.703-0.878<0.001^{*}$

0.864

$0.746-1.005 \quad 0.051$

$20<$ Tumor size $\leq 30$

\section{Gender}

Male

1

1

Female

0.669

$0.599-0.747<0.001^{*}$

0.828

$0.727-0.944$

$0.005^{\star}$

\section{Grade}

Well/moderate

1

Poor/UD

$1.4221 .262-1.602<0.001$ *

Unknown

$0.895 \quad 0.715-1.120 \quad 0.331$

* indicates that the difference was statistically significant

Abbreviations: UD, undifferentiated; SP, surgical procedure; SR,sublobar resection; LT, lobectomy; LNs, lymph nodes. 


\section{Adenocarcinoma}

\section{Resected LNs}

0

$1-3$

$\geq 4$

Unknown

Marriage status

Married

Unmarried

Others

Race

White

Black

Others

SP

SR

LT

* indicates that the difference was statistically significant

Abbreviations: UD, undifferentiated; SP, surgical procedure; SR,sublobar resection; LT, lobectomy; LNs, lymph nodes.

\section{Squamous cell carcinoma}

1

$0.664 \quad 0.552-0.799<0.001^{*} \quad 0.686 \quad 0.540-0.872 \quad 0.002^{*}$

$0.553 \quad 0.463-0.660<0.001 * \quad 0.644 \quad 0.513-0.809<0.001 *$

$\begin{array}{llllll}0.685 & 0.527-0.894 & 0.005^{*} & 1.309 & 0.943-1.818 & 0.107\end{array}$
$1.316 \quad 1.175-1.473<0.001 *$

$0.826 \quad 0.585-1.165 \quad 0.275$

1

$1.012 \quad 0.795-1.289 \quad 0.921$

$0.763 \quad 0.614-0.948 \quad 0.015^{\star}$

1

1
$0.742 \quad 0.624-0.883 \quad 0.001^{*}$
0.816
0.709-0.938 $0.004^{*}$ 
Table 5

Multivariate regression analyses for lung cancer specific survival stratified by tumor size

\section{Adenocarcinoma}

$\mathrm{HR} \quad 95 \% \mathrm{Cl}$
Squamous cell carcinoma

$\mathrm{HR} \quad 95 \% \mathrm{Cl}$

Tumor size $\leq 10(\mathrm{~mm})$

\section{Gender}

Male

1

Female

0.527

$0.346-0.802$

$0.003^{*}$

\section{Grade}

Well/moderate

1

$\begin{array}{llll}\text { Poor/UD } & 2.073 & 1.253-3.429 & 0.005^{*} \\ \text { Unknown } & 0.787 & 0.375-1.652 & 0.527\end{array}$

\section{Resected LNs}

0

1

$1-3$

$0.746 \quad 0.407-1.367 \quad 0.343$

$\geq 4$

$0.524 \quad 0.321-0.855 \quad 0.010$ *

Unknown

0.88

$0.391-2.017$

0.776

SP

SR

1

LT

$1.074 \quad 0.626-1.843$

0.795

$10<$ Tumor size $\leq 20$

Grade

Well/moderate

1

Poor/UD

1.951

$1.590-2.394$

$<0.001^{\star}$

Unknown

1.014

$0.721-1.425$

0.936

\section{Resected LNs}

0

* indicates that the difference was statistically significant

Abbreviations: UD, undifferentiated; SP, surgical procedure; SR, sublobar resection; LT, lobectomy; LNs, lymph nodes. 


\begin{tabular}{|c|c|c|c|c|c|c|}
\hline \multirow{2}{*}{$1-3$} & \multicolumn{3}{|c|}{ Adenocarcinoma } & \multicolumn{3}{|c|}{ Squamous cell carcinoma } \\
\hline & 0.853 & $0.646-1.126$ & 0.263 & & & \\
\hline$\geq 4$ & 0.617 & $0.468-0.813$ & $<0.001^{*}$ & & & \\
\hline Unknown & 0.390 & $0.231-0.661$ & $<0.001^{*}$ & & & \\
\hline \multicolumn{7}{|l|}{ Marriage status } \\
\hline Married & 1 & & & & & \\
\hline Unmarried & 1.179 & $0.988-1.406$ & 0.067 & & & \\
\hline Others & 0.906 & $0.529-1.552$ & 0.720 & & & \\
\hline \multicolumn{7}{|l|}{ Race } \\
\hline White & 1 & & & & & \\
\hline Black & 1.215 & $0.817-1.805$ & 0.336 & & & \\
\hline Others & 0.657 & $0.424-1.017$ & 0.060 & & & \\
\hline \multicolumn{7}{|l|}{ SP } \\
\hline SR & 1 & & & & & \\
\hline LT & 0.962 & $0.766-1.209$ & 0.741 & & & \\
\hline \multicolumn{7}{|c|}{$20<$ Tumor size $\leq 30$} \\
\hline \multicolumn{7}{|l|}{ Grade } \\
\hline Well/moderate & 1 & & & & & \\
\hline Poor/UD & 1.680 & $1.358-2.078$ & $<0.001^{*}$ & & & \\
\hline Unknown & 0.882 & $0.576-1.349$ & 0.562 & & & \\
\hline \multicolumn{7}{|l|}{ Resected LNs } \\
\hline 0 & 1 & & & 1 & & \\
\hline $1-3$ & 0.679 & $0.482-0.956$ & $0.027 *$ & 0.845 & $0.535-1.335$ & 0.470 \\
\hline$\geq 4$ & 0.516 & $0.370-0.719$ & $<0.001^{\star}$ & 0.771 & $0.497-0.991$ & $0.045^{\star}$ \\
\hline Unknown & 0.659 & $0.403-1.079$ & 0.108 & 1.519 & $0.818-2.822$ & 0.185 \\
\hline
\end{tabular}

* indicates that the difference was statistically significant

Abbreviations: UD, undifferentiated; SP, surgical procedure; SR, sublobar resection; LT, lobectomy; LNs, lymph nodes. 


SR 1
LT
0.954
$0.732-1.244$
0.729
0.776
$0.563-1.071$
0.123

* indicates that the difference was statistically significant

Abbreviations: UD, undifferentiated; SP, surgical procedure; SR, sublobar resection; LT, lobectomy; LNs, lymph nodes.

Additionally, we found that advanced tumor grade was strongly associated with worse OS (Multivariate: TS $\leq 10 \mathrm{~mm}: \mathrm{HR}=1.354,95 \% \mathrm{Cl}(1.047,1.750), \mathrm{P}=0.021$; $\mathrm{TS}>10 \mathrm{~mm} \& \leq 20 \mathrm{~mm}: \mathrm{HR}=1.582,95 \% \mathrm{Cl}$ (1.422 1.761), $\mathrm{P}<0.001 ; \mathrm{TS}>20 \mathrm{~mm} \& \leq 30 \mathrm{~mm}: \mathrm{HR}=1.422,95 \% \mathrm{Cl}(1.262,1.602), \mathrm{P}<0.001)$ and LCSS (Multivariate: $\mathrm{TS} \leq 10 \mathrm{~mm}: \mathrm{HR}=2.073,95 \% \mathrm{Cl}(1.253,3.429), \mathrm{P}=0.003 ; \mathrm{TS}>10 \mathrm{~mm} \& \leq 20 \mathrm{~mm}: \mathrm{HR}=$ $1.951,95 \% \mathrm{Cl}(1.590,2.394), \mathrm{P}<0.001$; TS $>20 \mathrm{~mm} \& \leq 30 \mathrm{~mm}: \mathrm{HR}=1.680,95 \% \mathrm{Cl}(1.358,2.078), \mathrm{P}<$ 0.001 ) in LUAD patients; however, in LSQCC patients, the difference not observed in both OS (Univariate: $\mathrm{TS} \leq 10 \mathrm{~mm}: \mathrm{HR}=1.116,95 \% \mathrm{Cl}(0.847,1.470), \mathrm{P}=0.436 ; \mathrm{TS}>10 \mathrm{~mm} \& \leq 20 \mathrm{~mm}: \mathrm{HR}=1.088,95 \% \mathrm{Cl}$ $(0.966,1.225), \mathrm{P}=0.164 ; \mathrm{TS}>20 \mathrm{~mm} \& \leq 30 \mathrm{~mm}: \mathrm{HR}=0.932,95 \% \mathrm{Cl}(0.817,1.062), \mathrm{P}=0.291)$ and LCSS (Univariate: $\mathrm{TS} \leq 10 \mathrm{~mm}: \mathrm{HR}=1.423,95 \% \mathrm{Cl}(0.763,2.653), \mathrm{P}=0.268 ; \mathrm{TS}>10 \mathrm{~mm} \& \leq 20 \mathrm{~mm}: \mathrm{HR}=1.291$, $95 \% \mathrm{Cl}(1.000,1.658), \mathrm{P}=0.050 ; \mathrm{TS}>20 \mathrm{~mm} \& \leq 30 \mathrm{~mm}: \mathrm{HR}=0.994,95 \% \mathrm{Cl}(0782,1.264), \mathrm{P}=0.964)$ (Data shown in Supplementary table 3 and 4 ).

Moreover, multivariate analyses demonstrated that a larger number of resected $\mathrm{LNs}$ predicted favorable OS and LCSS in LUAD with TS $\leq 30 \mathrm{~mm}$ and LSQCC with TS $>10 \mathrm{~mm} \& \leq 30 \mathrm{~mm}$ (Data shown in Tables 4 and 5); while in LSQCC, the differences were not significant in TS $\leq 10 \mathrm{~mm}$ (Univariate: OS: "1-3" vs "0": $\mathrm{HR}=0.833,95 \% \mathrm{Cl}(0.573,1.210), \mathrm{P}=0.337$; “ $\geq 4$ " vs. "0", $\mathrm{HR}=0.843,95 \% \mathrm{Cl}(0.626,1.134), \mathrm{P}=0.258$; LCSS: " $1-3$ " vs “0” $\mathrm{HR}=1.446,95 \% \mathrm{Cl}(0.601,3.476), \mathrm{P}=0.410$; “ $\geq 4$ ” vs. “0”, $\mathrm{HR}=1.205,95 \% \mathrm{Cl}(0.564$, 2.577), $P=0.630$ ) (Data shown in Supplementary table 3 and 4).

\section{Discussion}

In present study, we investigated the impact of histology on prognosis of elderly patients with surgically resected IA stage LUAD and LSQCC. Univariate analyses showed that LT closely was associated with better OS $(\mathrm{HR}=0.647,95 \% \mathrm{Cl}(0.606,0.689), \mathrm{P}<0.001)$ and LCSS $(\mathrm{HR}=0.834,95 \% \mathrm{Cl}(0.731,0.951), \mathrm{P}=$ $0.007)$ in $\triangle A$ stage LUAD patients; while in LSQCC, LT yielded better OS $(H R=0.706,95 \% \mathrm{Cl}(0.649,0.769)$, $\mathrm{P}<0.001)$ but not better LCSS $(\mathrm{HR}=0.884,95 \% \mathrm{Cl}(0.740,1.055), \mathrm{P}=0.171)$. Multivariate analyses demonstrated that $\mathrm{LT}$ achieved better OS (HR $=0.806,95 \% \mathrm{Cl}(0.743,0.873), \mathrm{P}<0.001)$ but not LCSS (HR = $0.992,95 \% \mathrm{Cl}(0.841,1.170), \mathrm{P}=0.920)$ in LUAD patients compared with SR. In LSQCC patients, LT was associated with better OS $(\mathrm{HR}=0.804,95 \% \mathrm{Cl}(0.722,0.895), \mathrm{P}<0.001)$ in patients compared with SR.

Recently, numerous studies pointed out that LSQCC and LUAD patients are significantly different in many prognostic factors, including age, sex, pathological and clinical TNM stage, smoking status, tumor 
location, tumor differentiation grade and visceral pleura invasion[4, 18-20]. However, the prognosis of the two types of lung cancer had mixed results. Previous studies suggested that LSQCC had a favorable survival compared to LUAD[21, 22]. Some researchers found that there was no significant difference in prognosis between LSQCC and LUAD[23, 24]. However, recent studies demonstrated that LUAD was associated with better survival compared to $\operatorname{LSQCC}[5,6,25]$. Although more and more people came to realize that two types of lung cancer should be separately analyzed, few studies focused on investigating whether the different types of histology could have an impact on outcome of elderly patients with earlystage NSCLC. Furthermore, the question whether the limited resection is suitable for early-stage NSCLC remains to be investigating, and lobectomy is still the standard treatment for early-stage NSCLC according to National Comprehensive Cancer Network (NCCN) guidelines. However, there is emerging evidence from retrospective studies supporting that subloar resection suitable for certain early-stage NSCLC[26-29]. In our study, we found that SR yielded similar OS compared to LT for LUAD patients with TS $\leq 10 \mathrm{~mm}$, and for LSQCC patients with TS $\leq 20 \mathrm{~mm}$, LT didn't show superior OS compared to SR. For LUAD patients with TS $>10 \mathrm{~mm} \& \leq 30 \mathrm{~mm}$, LT achieved better OS compared to SR; while for LSQCC patients with TS > $20 \mathrm{~mm}$ $\& \leq 30 \mathrm{~mm}$, LT achieved better OS compared to SR. However, LT yielded similar LCSS in both elderly LUAD and LSQCC patients with TS $\leq 30 \mathrm{~mm}$. These results suggested that SR maybe acceptable for elderly LUAD patients with TS $\leq 10 \mathrm{~mm}$ and LSQCC patients with TS $\leq 20 \mathrm{~mm}$. As mentioned, elderly patients were likely to have declined cardiopulmonary reserve and a limited life expectancy, and limited resections reduced lung function impairment and might reduce morbidity. The high-quality randomized controlled trials, including the Japan Clinical Oncology Group/West Japan Oncology Group (JCOG0802/WJOG4607L) and the Cancer and Lymphoma Group (CALBG 140503), were ongoing. Our results need to be verified in the future.

In addition, we found that advanced tumor grade was significantly associated with poorer OS and LCSS in elderly LUAD patients; while in LSQCC, the difference was not significant. We speculated that early stage LSQCC was likely to have less metastasis tendency than LUAD. Consistent with our speculation, Wang et al[5]. pointed out that LSQCC was mostly found in stage II and IIIA, whereas LUAD was mostly found in stage I. They speculated that LUAD patients tended to have an earlier clinical T status than LSQCC patients but have stronger metastasis tendency. The reason for that may be the different mechanisms of metastasis. Cao et al. [30] found that a larger number of resected regional LNs predicted better LCSS and OS rates compared with less regional LN dissection. Moreover, we also observed that a larger number of resected lymph nodes (LNs) was strongly associated with better OS and LCSS, and, however, the difference was not observed in LSQCC patients with TS $\leq 10 \mathrm{~mm}$, which may support our speculation that early stage LSQCC was likely to have less lymph nodes metastasis tendency than LUAD. For LSQCC patients with TS $>10 \mathrm{~mm}$ and LUAD patients, mediastinal lymph nodes evaluation should be paid attention.

The limitations of this study include the lack of targeted therapy information and immunotherapy information as well as the nature of retrospective studies. The impact of the former factor is limited because of the lower chance for these early stage patients to receive such treatments; while that of the latter is inevitable. Multivariate analyses were conducted to remove biases as could as possible. There is 
no detailed information about pure ground-glass opacity (GGO), mixed GGO, and pure solid adenocarcinoma as well as lack of information regarding the status of tumor component; thus, we could not take these factors into consideration. In addition, micropapillary was recently reported as an important prognostic factor that was closely related to the spread of lung cancer tumor cells into air spaces in the lung parenchyma adjacent to the main tumor[31]. However, the information about micropapillary couldn't be obtained in SEER database. Thus, our conclusions still need to be further verified in future large randomized cohort studies.

\section{Conclusions}

In conclusion, we found that LUAD patients with age $>70$ had superior OS and LCSS compared to that of LSQCC. We also observed that a larger number of resected LNs was associated with favorable OS and LCSS in LUAD with TS $\leq 30 \mathrm{~mm}$ and LSQCC with TS $>10 \mathrm{~mm} \& \leq 30 \mathrm{~mm}$. Our study demonstrated that SR achieved similar OS in LUAD patients with TS $\leq 10 \mathrm{~mm}$ and LSQCC patients with TS $\leq 20 \mathrm{~mm}$ compared to $L T$, which suggested that SR maybe acceptable for elderly LUAD patients with TS $\leq 10 \mathrm{~mm}$ and LSQCC patients with $\mathrm{TS} \leq 20 \mathrm{~mm}$.

\section{Abbreviations}

LUAD, lung adenocarcinoma; LSQCC, lung squamous cell carcinoma; NSCLC, non-small lung cancer; SEER, Surveillance, Epidemiology, and End Results; OS, overall survival; LCSS, lung cancer-specific survival; TS, tumor size; LT, lobectomy; SR, sublobar resection; SP, surgical procedure; $\mathrm{HR}$, hazard ratio; $\mathrm{Cl}$, confidence interval; LN, lymph node.

\section{Declarations}

\section{Acknowledgements}

We acknowledged the SEER database for providing clinical data.

\section{Author contributions}

BH: Study design, data analysis, manuscript preparation, and final approve of manuscript

HJF: Study design, data analysis and revision of the manuscript.

XXW: Study design, data analysis and revision of the manuscript.

LZ: Data analysis and revision of the manuscript.

ZLL: Data analysis and revision of the manuscript.

BHL: Data analysis and revision of the manuscript. 
RYH: Data analysis and revision of the manuscript.

HM: Data analysis and revision of the manuscript.

TF: Data analysis and revision of the manuscript.

WYJ: Data analysis and revision of the manuscript.

QG: Study design, data analysis, revision of the manuscript and final approve of manuscript

\section{Funding}

The work was funded by the National Natural Science Foundation of China (grant no. 81700093 and 81770095). The funding bodies had no role in the design of the study, the collection, analysis, or interpretation of the data, or writing the manuscript.

\section{Availability of data and materials}

All data in the present study is available in the SEER database (https://seer.cancer.gov/ data/).

\section{Ethics approval and consent to participate}

Not appliable

\section{Consent for publication}

All authors approved for publication of the manuscript for the journal.

\section{Declaration of Competing interest}

All of the authors declare no conflicts of interest for this paper.

\section{Author details}

1 Department of Thoracic Surgery, Renmin Hospital of Wuhan University, Wuhan, China.

2 Department of Thoracic Surgery, Affiliated Ezhou Central Hospital, Wuhan University, Ezhou, China.

\section{References}

1. Torre LA, Siegel RL, Jemal A. Lung Cancer Statistics. Adv Exp Med Biol. 2016;893:1-19.

2. Choi CM, Kim HC, Jung CY, Cho DG, Jeon JH, Lee JE, Ahn JS, Kim SJ, Kim Y, Choi YD, et al. Report of the Korean Association of Lung Cancer Registry (KALC-R), 2014. Cancer Res Treat. 2019;51:1400-10. 
3. Kawaguchi T, Takada M, Kubo A, Matsumura A, Fukai S, Tamura A, Saito R, Maruyama Y, Kawahara $\mathrm{M}$, Ignatius Ou SH. Performance status and smoking status are independent favorable prognostic factors for survival in non-small cell lung cancer: a comprehensive analysis of 26,957 patients with NSCLC. J Thorac Oncol. 2010;5:620-30.

4. Chansky K, Sculier JP, Crowley JJ, Giroux D, Van Meerbeeck J, Goldstraw P, International Staging C, Participating I. The International Association for the Study of Lung Cancer Staging Project: prognostic factors and pathologic TNM stage in surgically managed non-small cell lung cancer. J Thorac Oncol. 2009;4:792-801.

5. Wang BY, Huang JY, Chen HC, Lin CH, Lin SH, Hung WH, Cheng YF. The comparison between adenocarcinoma and squamous cell carcinoma in lung cancer patients. J Cancer Res Clin Oncol. 2020;146:43-52.

6. Nakamura H, Sakai H, Kimura H, Miyazawa T, Marushima H, Saji H. Difference in Postsurgical Prognostic Factors between Lung Adenocarcinoma and Squamous Cell Carcinoma. Ann Thorac Cardiovasc Surg. 2017;23:291-7.

7. Allen MS, Darling GE, Pechet TT, Mitchell JD, Herndon JE 2nd, Landreneau RJ, Inculet RI, Jones DR, Meyers BF, Harpole DH,et al. Morbidity and mortality of major pulmonary resections in patients with early-stage lung cancer: initial results of the randomized, prospective ACOSOG Z0030 trial. Ann Thorac Surg. 2006;81:1013-9. discussion 1019-1020.

8. Okami J, Higashiyama M, Asamura H, Goya T, Koshiishi Y, Sohara Y, Eguchi K, Mori M, Nakanishi Y, Tsuchiya R,et al. Pulmonary resection in patients aged 80 years or over with clinical stage I non-small cell lung cancer: prognostic factors for overall survival and risk factors for postoperative complications. J Thorac Oncol. 2009;4:1247-53.

9. Liu Y, Shan L, Shen J, Liu L, Wang J, He J, He Q, Jiang L, Guo M, Chen X, et al. Choice of surgical procedure - lobectomy, segmentectomy, or wedge resection - for patients with stage T1-2N0M0 small cell lung cancer: A population-based study. Thorac cancer. 2019;10:593-600.

10. Dai C, Shen J, Ren Y, Zhong S, Zheng H, He J, Xie D, Fei K, Liang W, Jiang G, et al. Choice of Surgical Procedure for Patients With Non-Small-Cell Lung Cancer $</=1 \mathrm{~cm}$ or $>1$ to $2 \mathrm{~cm}$ Among Lobectomy, Segmentectomy, and Wedge Resection: A Population-Based Study. J Clin Oncol.2016; 34:3175-3182.

11. Moon MH, Moon YK, Moon SW. Segmentectomy versus lobectomy in early non-small cell lung cancer of $2 \mathrm{~cm}$ or less in size: A population-based study. Respirology. 2018;23:695-703.

12. Soneji S, Tanner NT, Silvestri GA, Lathan CS, Black W. Racial and Ethnic Disparities in Early-Stage Lung Cancer Survival. Chest. 2017;152:587-97.

13. Yoon JY, Sigel K, Martin J, Jordan R, Beasley MB, Smith C, Kaufman A, Wisnivesky J, Kim MK. Evaluation of the Prognostic Significance of TNM Staging Guidelines in Lung Carcinoid Tumors. J Thorac Oncol. 2019;14:184-92.

14. Thakur MK, Ruterbusch JJ, Schwartz AG, Gadgeel SM, Beebe-Dimmer JL, Wozniak AJ. Risk of Second Lung Cancer in Patients with Previously Treated Lung Cancer: Analysis of Surveillance, Epidemiology, and End Results (SEER) Data. J Thorac Oncol. 2018;13:46-53. 
15. Wang Y, Pang Z, Chen X, Bie F, Wang Y, Wang G, Liu Q, Du J. Survival nomogram for patients with initially diagnosed metastatic non-small-cell lung cancer: a SEER-based study. Future Oncol. 2019;15:3395-409.

16. Morgensztern D, Waqar S, Subramanian J, Gao F, Trinkaus K, Govindan R. Prognostic significance of tumor size in patients with stage III non-small-cell lung cancer: a surveillance, epidemiology, and end results (SEER) survey from 1998 to 2003. J Thorac Oncol. 2012;7:1479-84.

17. Zhao M, Lu T, Huang Y, Yin J, Jiang T, Li M, Yang X, Zhan C, Feng M, Wang Q. Survival and Long-Term Cause-Specific Mortality Associated With Stage IA Lung Adenocarcinoma After Wedge Resection vs. Segmentectomy: A Population-Based Propensity Score Matching and Competing Risk Analysis. Front Oncol. 2019;9:593.

18. Qiu C, Wang G, Xu J, Cui L, Dong W, Ni Y, Qu X, Du J. Sublobectomy versus lobectomy for stage I nonsmall cell lung cancer in the elderly. Int J Surg. 2017;37:1-7.

19. Kawase A, Yoshida J, Ishii G, Nakao M, Aokage K, Hishida T, Nishimura M, Nagai K. Differences between squamous cell carcinoma and adenocarcinoma of the lung: are adenocarcinoma and squamous cell carcinoma prognostically equal? Jpn J Clin Oncol. 2012;42:189-95.

20. Usui S, Minami Y, Shiozawa T, lyama S, Satomi K, Sakashita S, Sato Y, Noguchi M. Differences in the prognostic implications of vascular invasion between lung adenocarcinoma and squamous cell carcinoma. Lung Cancer. 2013;82:407-12.

21. Wisnivesky JP, Henschke C, McGinn T, lannuzzi MC. Prognosis of Stage II non-small cell lung cancer according to tumor and nodal status at diagnosis. Lung Cancer. 2005;49:181-6.

22. Strand TE, Rostad H, Moller B, Norstein J. Survival after resection for primary lung cancer: a population based study of 3211 resected patients. Thorax. 2006;61:710-5.

23. Jemal A, Clegg LX, Ward E, Ries LA, Wu X, Jamison PM, Wingo PA, Howe HL, Anderson RN, Edwards BK. Annual report to the nation on the status of cancer, 1975-2001, with a special feature regarding survival. Cancer.2004; 101:3-27.

24. Puri V, Garg N, Engelhardt EE, Kreisel D, Crabtree TD, Meyers BF, Patterson GA, Krupnick AS. Tumor location is not an independent prognostic factor in early stage non-small cell lung cancer. Ann Thorac Surg. 2010;89:1053-9.

25. Fukui T, Taniguchi T, Kawaguchi K, Fukumoto K, Nakamura S, Sakao Y, Yokoi K. Comparisons of the clinicopathological features and survival outcomes between lung cancer patients with adenocarcinoma and squamous cell carcinoma. Gen Thorac Cardiovasc Surg. 2015;63:507-13.

26. Zeng W, Zhang W, Zhang J, You G, Mao Y, Xu J, Yu D, Peng J, Wei Y. Systematic review and metaanalysis of video-assisted thoracoscopic surgery segmentectomy versus lobectomy for stage I nonsmall cell lung cancer. World J Surg Oncol. 2020;18:44.

27. Sienel W, Dango S, Kirschbaum A, Cucuruz B, Horth W, Stremmel C, Passlick B. Sublobar resections in stage IA non-small cell lung cancer: segmentectomies result in significantly better cancer-related survival than wedge resections. Eur J Cardiothorac Surg. 2008;33:728-34. 
28. Scott WJ, Howington J, Feigenberg S, Movsas B, Pisters K, American College of Chest P. Treatment of non-small cell lung cancer stage I and stage II: ACCP evidence-based clinical practice guidelines (2nd edition). Chest.2007; 132:234S-242S.

29. Altorki NK, Yip R, Hanaoka T, Bauer T, Aye R, Kohman L, Sheppard B, Thurer R, Andaz S, Smith M,et al. Sublobar resection is equivalent to lobectomy for clinical stage $1 \mathrm{~A}$ lung cancer in solid nodules. $\mathrm{J}$ Thorac Cardiovasc Surg. 2014;147:754-62. Discussion 762 - 754.

30. Cao J, Xu J, He Z, Yuan P, Huang S, Lv W, Hu J. Prognostic impact of lymphadenectomy on outcomes of sublobar resection for stage IA non-small cell lung cancer $</=2 \mathrm{~cm}$. J Thorac Cardiovasc Surg. 2018;156:796-805 e794.

31. Kadota K, Nitadori JI, Sima CS, Ujiie H, Rizk NP, Jones DR, Adusumilli PS, Travis WD. Tumor Spread through Air Spaces is an Important Pattern of Invasion and Impacts the Frequency and Location of Recurrences after Limited Resection for Small Stage I Lung Adenocarcinomas. J Thorac Oncol. 2015;10:806-14.

\section{Figures}
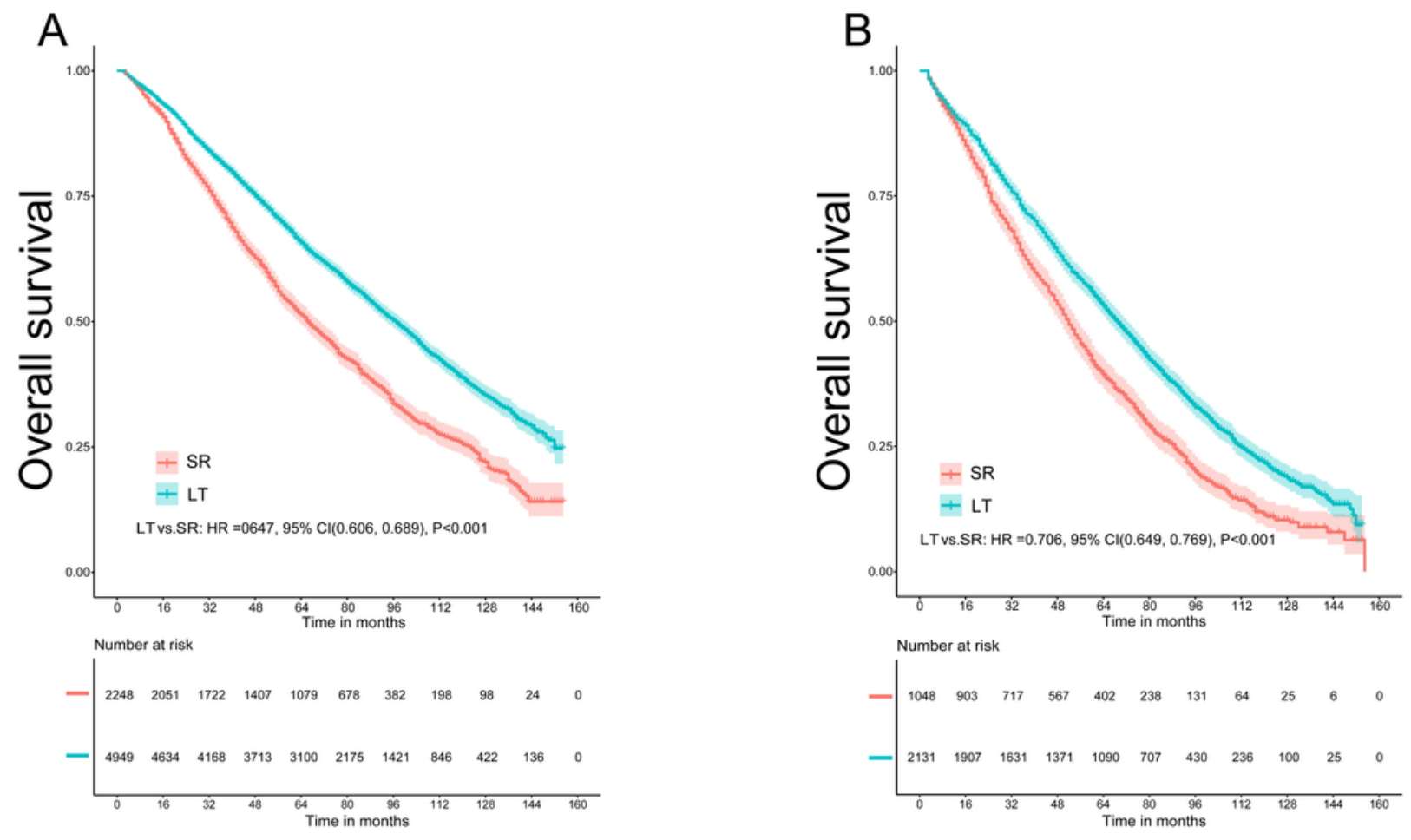

\section{Figure 1}

Postoperative overall survival curves between sublobar resection (SR) and lobectomy (LT). 
A
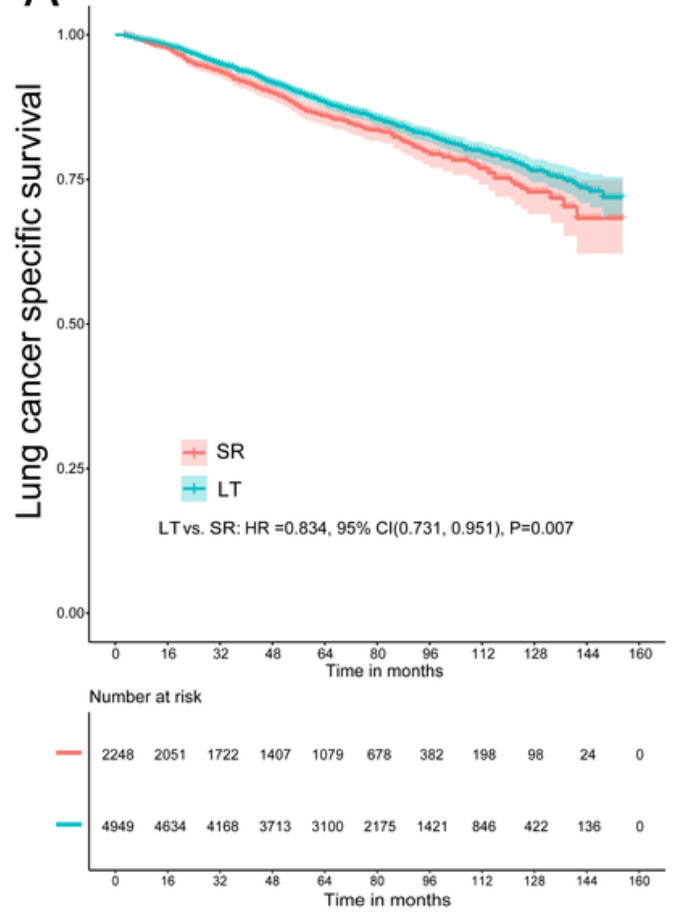

B
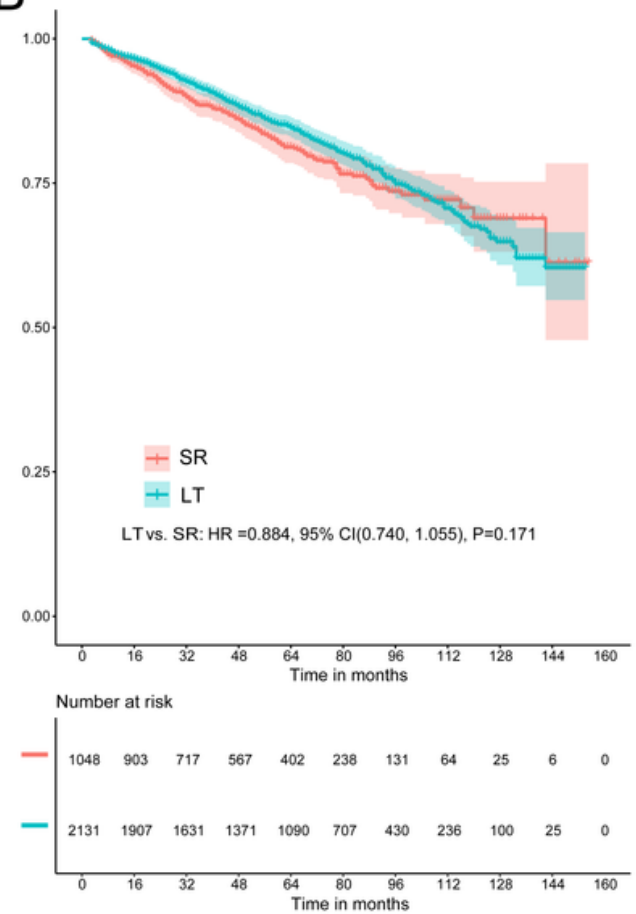

Figure 2

Postoperative lung cancer specific survival curves between sublobar resection (SR) and lobectomy (LT).

\section{Supplementary Files}

This is a list of supplementary files associated with this preprint. Click to download.

- Supplementaryfigure1.tif

- Supplementaryfigure2.tif

- Supplementarytable1.docx

- Supplementarytable2.docx

- Supplementarytable3.docx

- Supplementarytable4.docx 